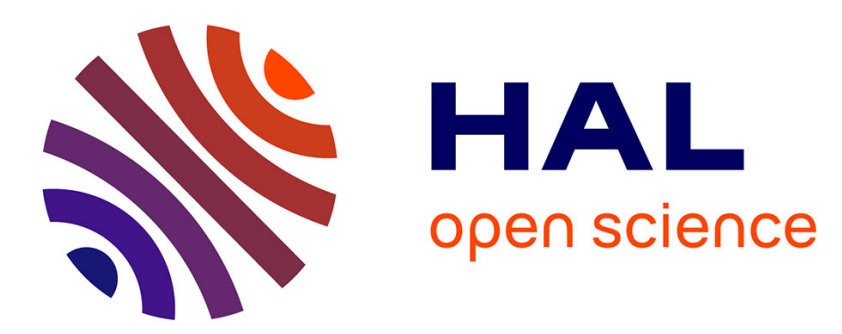

\title{
Augmentative biocontrol when natural enemies are subject to Allee effects
}

Nicolas Bajeux, Frédéric Grognard, Ludovic Mailleret

\section{To cite this version:}

Nicolas Bajeux, Frédéric Grognard, Ludovic Mailleret. Augmentative biocontrol when natural enemies are subject to Allee effects. Journal of Mathematical Biology, 2017, 74 (7), pp.1561 - 1587. 10.1007/s00285-016-1063-8. hal-01402250v2

\section{HAL Id: hal-01402250 \\ https://hal.science/hal-01402250v2}

Submitted on 14 Mar 2022

HAL is a multi-disciplinary open access archive for the deposit and dissemination of scientific research documents, whether they are published or not. The documents may come from teaching and research institutions in France or abroad, or from public or private research centers.
L'archive ouverte pluridisciplinaire HAL, est destinée au dépôt et à la diffusion de documents scientifiques de niveau recherche, publiés ou non, émanant des établissements d'enseignement et de recherche français ou étrangers, des laboratoires publics ou privés. 


\title{
Augmentative biocontrol when natural enemies are subject to Allee effects
}

\author{
Nicolas Bajeux · Frédéric Grognard · Ludovic \\ Mailleret
}

Received: date / Accepted: date

\begin{abstract}
Intraspecific interactions such as Allee effects are key properties that can guide population management. This contribution considers component Allee effects that are elementary mechanisms leading to declines of fitness at the population scale, i.e. demographic Allee effects. It especially focuses on the consequences of such properties in predator populations, and investigates their repercussions in a biological control context. A modelling framework able to account for reproductive and/or foraging component Allee effects is proposed. From this, four models of augmentative biological control, corresponding to the periodic introduction of natural enemies, have been investigated. This is done using semi-discrete models: ordinary differential equations are used to depict predator-prey dynamics and a discrete equation describes the abrupt augmentation of predators at periodic intervals.

In that context, stability of a prey-free solution corresponding to pest eradication has been analyzed. It has been found that rare but large introductions should be preferred over frequent and small ones, when Allee effects influence predator populations. In particular, the occurrence of foraging, rather than reproducing, Allee effects significantly hinders pest eradication. Cases where the pest-free solution is locally, but not globally, stable were also observed and were shown to be favored by the occurrence of reproductive Allee effects among predators.
\end{abstract}

Keywords Predator-prey models $\cdot$ positive density dependence $\cdot$ impulsive differential equations $\cdot$ semi-discrete models $\cdot$ stabilization.

N. Bajeux · F. Grognard · L. Mailleret

INRIA Sophia Antipolis-Méditerranée, BIOCORE, France

E-mail: nicolas.bajeux@inria.fr

F. Grognard

E-mail: frederic.grognard@inria.fr

L. Mailleret

INRA, Univ. Nice Sophia Antipolis, CNRS, UMR 1355-7254, Institut Sophia Agrobiotech, 06900 Sophia Antipolis, France

E-mail: ludovic.mailleret@sophia.inra.fr 


\section{Introduction}

Crop pests are organisms that have harmful impacts on cultivated plants. Pests generate damages to agricultural crops by feeding on (herbivores), parasitizing (pathogens) or competing (weeds) with crops. The presence of such organisms generally leads to crop losses (Oerke, 2006). A simple and quite effective way to regulate these harmful organisms is to use chemical pesticides, a method which is massively used for crop protection worldwide (Pimentel et al, 1992, Wilson and Tisdell, 2001). However, such chemical control can negatively impact crops as well as ecosystems surrounding them. Pesticides indeed affect every living organism in contact with them (Pimentel and Edwards, 1982, McLaughlin and Mineau, 1995). Such methods also have harmful impacts on farmers and consumers health (Bolognesi and Morasso, 2000). Fortunately, alternative controls exist to reduce or prevent damages caused by pest populations: cultural control which changes cropping habits such as polyculture or crop rotation, physical pest control with barriers or traps for example, mechanical pest control by using handpicking or weeding or biological control, which relies on the use of natural enemies such as predators, parasitoids or parasites so as to reduce or suppress the targeted harmful organism (DeBach and Rosen, 1991).

There exist three main biological control methods: (i) conservation biological control which consists in improving the environment for local natural enemies, (ii) classical biological control which is based on the introduction of a new enemy when it is not native of the region or has been removed previously and (iii) augmentation biological control which relies on repeated releases of mass-reared natural enemies. In the latter case, natural enemies can be released inoculatively, using periodic introductions to exert long-term regulation of the pest or inundatively, meaning that a mass amount of enemies are released at each introduction in order to kill pests quickly (Lacey et al, 2001). Such techniques are extensively used in greenhouse pest control programs (Van Lenteren, 2000, Collier and Steenwyk, 2004). For instance, parasitoids of the genus Trichogramma are used in this way to control arthropods (Van Lenteren, 2000).

The difficulty of augmentative biological control is to identify how many and how frequently natural enemies should be introduced to achieve pest eradication. In invasion and conservation biology, the combination of the frequency of introductions and the number of individual introduced each time is called propagule pressure or introduction effort (Simberloff, 2009). It concerns both human-mediated and natural invasions. Propagule pressure takes into account two components: the propagule size (number of released individuals), and the propagule number (number or frequency of introduction events (Wittmann et al, 2014)). Studying how the components of propagule pressure influence population establishment and/or the services they provide, e.g. biological control, is an important challenge of both experimental and theoretical research on introduced populations (Simberloff. 2009: Wittmann et al, 2014). From the latter point of view, the presence of repeated introductions of individuals sets a scene where continuous-time population dynamics are disrupted by jumps in the introduced population density. To handle this, a classical approach is to use semi-discrete models which combine ordinary differential equations to depict population dynamics with 
discrete-time difference equations to describe introductions (Mailleret and Lemesle 2009).

To improve augmentative biological control, it is also important to better understand inter- and intra-specific interactions that occur once predators have been released into the targeted area. For instance, negative density dependence such as competition among predators have been studied in augmentative biological control. Modeling studies recommended to rather inoculate predators frequently in small quantity than to inundate the area (Nundloll et al, 2010b a). Another form of intra-specific interactions is positive density dependence of the growth rate of the population, i.e. Allee effects. This phenomenon has been first described by W.C. Allee who showed that cooperation between individuals does lead to positive density dependence (Allee 1949). (Stephens and Sutherland, 1999) classifies Allee effects as 'component' and 'demographic' Allee effects. A component Allee effect is defined as a positive relationship between one component of individual fitness and population size or density. A demographic Allee effect refers to a positive relationship between the mean fitness of the population, i.e. its per capita growth rate, and population size or density.

Various mechanisms generate component Allee effects which may, or may not, lead to demographic Allee effects (Courchamp et al, 2008). Mechanisms linked to reproduction are reported, such as the mate-finding Allee effect which is the difficulty to find mates when populations are small (Gascoigne et al, 2009; Boukal and Berec, 2009). Cooperative breeding makes it difficult for a social population to breed offspring at low population size (Courchamp and Macdonald, 2001). There are also mechanisms related to survival such as cooperative anti-predator behavior or alterations of the foraging efficiency resulting from the difficulty to locate or capture resources when the population is small (Courchamp et al, 2008, Berec et al, 2007).

Although the importance of Allee effects for population management is increasingly recognized (Derederec and Courchamp, 2007, Liebhold and Tobin, 2008), theoretical investigations of the consequences of Allee effects among predator populations remain quite rare (Bazykin, 1998, Zhou et al, 2005, Verdy, 2010, Terry, 2015). This observation is also valid for the broader category of "biological control agents" and theory on the influence of Allee effects in the natural enemies on the success of biological control is scarce (but see (Bompard et al, 2013)). Yet, component Allee effects have been reported on diverse parasitoid or predator species that can serve as biological control agents. This is for instance the case of the parasitoid Aphelinus asychis (Walker) which is used against Diuraphis noxia (Mordvilko) (Fauvergue and Hopper, 2009). On the conservation biological control side, the barn swallow Hirundo rustica, is an insectivore bird subject to sexual selection that can lead to demographic Allee effects (Møller and Legendre, 2001; Møller and Gregersen, 1994).

In the present work, we provide a set of models generalizing those proposed by (Verdy, 2010) using generic functions to represent predator-prey dynamics. The different models we propose will take into account different component Allee effects among the predator population as well as their combination. These models will then serve to examine the efficacy of augmentative biological control strategies and determine those ensuring pest eradication despite the positive density dependence. The respective impacts of the different component Allee effects that are considered will be discussed. 


\section{One model to rule them all}

\subsection{Predator-prey interactions}

A common framework to represent pest (prey) - natural enemies (predators) interactions is to use a system of two ODEs: the first one describes the prey dynamics and the other one models predator dynamics:

$$
\left\{\begin{array}{l}
\dot{x}=f(x)-g(x) y \\
\dot{y}=h(g(x)) y-d(g(x)) y
\end{array}\right.
$$

with $x$ and $y$ the prey and predator density, respectively. Intrinsic prey growth is modeled with function $f($.$) and g($.$) and h($.$) the functional and numerical responses of$ the predator, respectively. Although the proportionality between the functional and numerical response of the predator is a widespread assumption, there are numerous situations in which prey captures do not translate directly in predator births. This assumption is replaced by the less strict one that the functional response simply depends on prey captures per predator, i.e. the functional response. This is taken into account through the form $h(g(x))$ of the numerical response. Predator death rate is modeled through function $d($.$) .$

Classical assumptions are proposed for these functions: in absence of individuals, prey growth can not take place $(f(0)=0)$ and as prey invade, their population increases $\left(f^{\prime}(0)>0\right)$.

Without prey, prey uptake by predators can not occur $(g(0)=0)$, but it becomes immediately positive as prey are present $\left(g^{\prime}(0)>0\right.$ and $\left.\forall x>0, g(x)>0\right)$. The ratio of pest growth to functional response is supposed upper-bounded for $x \geq 0$, by $S$ such that $S=\sup _{x \geqslant 0}\left(\frac{f(x)}{g(x)}\right)$; it means that pest growth can not overwhelm predation, i.e. that when predators are sufficiently numerous they can suppress the prey population.

The numerical response is assumed to be increasing in prey captures, with the additional property that the absence of prey captures prevents births $(h(0)=0)$.

Following (Terry, 2014b), it is assumed that the predator mortality rate may be affected by the number of prey captures per predator $g(x)$ since starving predators may indeed be more prone to death; this is accounted for through the formulation $d(g(x))$, with $d($.$) a non-increasing function of its argument.$

In absence of prey, the mortality rate is maximum, its value is $d(0)=d_{0}$.

\subsection{Component Allee effects}

Model (1) does not describe intraspecific interactions in the predator population, although natural enemies may be subject to many of them. For instance, natural enemies may undergo Allee effects due to different causes. In this contribution, we studied the influence of two component Allee effects and their interaction: (i) one linked to reproductive mechanisms and (ii) the other linked to a decrease in foraging 


\begin{tabular}{ll}
\hline Function & Hypothesis \\
\hline$f(x)$ & $f(0)=0$ and $f^{\prime}(0)>0$ \\
$g(x)$ & $g(0)=0, g^{\prime}(0)>0$ and $\forall x>0, g(x)>0$ \\
$\frac{f(x)}{g(x)}$ & upper bounded, with $S=\sup _{x \geqslant 0}\left(\frac{f(x)}{g(x)}\right)$ \\
$h(z)$ & $h(0)=0$ and $\forall z>0, h(z) \geqslant 0$ and $h^{\prime}(z) \geqslant 0, \forall z \geqslant 0$ \\
$d(z)$ & $d(0)=d_{0}, d(z)>0$ and $d^{\prime}(z) \leqslant 0, \forall z \geqslant 0$ \\
$q_{f}(y)$ & $q_{f}(0)=q_{f_{0}}, q_{f}(y) \in[0,1], q_{f}^{\prime}(y) \geq 0$ and $\lim _{y \rightarrow+\infty} q_{i}(y)=1$ \\
$q_{r}(y)$ & same hypotheses as $q_{f}(y)$ \\
\hline
\end{tabular}

Table 1 Summary of hypotheses on the functions involved in the models. All functions are assumed to be of class $\mathscr{C}^{1}$ on $\mathbb{R}_{+}^{*}$.

efficiency at low population sizes (Table 2.1 in (Courchamp et al, 2008) lists mechanisms responsible for Allee effects). The latter component Allee effect can actually impact both predator reproduction and predator survival.

The dependency of predators foraging in predator density (mechanism (ii)) requires to modify the formulation of the functional response in model (1). It was simply done by modulating $g(x)$ by a function $q_{f}(y)$, which is non-decreasing in $y$. The model becomes:

$$
\left\{\begin{array}{l}
\dot{x}=f(x)-g(x) q_{f}(y) y, \\
\dot{y}=h\left(g(x) q_{f}(y)\right) y-d\left(g(x) q_{f}(y)\right) y .
\end{array}\right.
$$

Predators foraging efficiency is at its lower value when there is no predator: $q_{f}(0)=$ $q_{f_{0}}$. Note that $q_{f_{0}}$ is between 0 and 1 . When the predator population grows large, the component Allee effect vanishes so that $\lim _{y \rightarrow+\infty} q_{f}(y)=1$. Overall, smaller $q_{f_{0}}$ correspond to stronger Allee effects.

Strictly reproductive Allee effects (mechanism (i)) only appears in predator birth rates. Similarly to what has been done for the foraging mechanism, a function $q_{r}(y)$ is introduced to modulate the numerical response:

$$
\left\{\begin{array}{l}
\dot{x}=f(x)-g(x) y, \\
\dot{y}=h(g(x)) q_{r}(y) y-d(g(x)) y .
\end{array}\right.
$$

Function $q_{r}(y)$ has the very same mathematical form as $q_{f}(y)$.

Predator populations can be subject to one or the other component Allee effects, but also to both of them (Berec et al, 2007). The corresponding model reads:

$$
\left\{\begin{array}{l}
\dot{x}=f(x)-g(x) q_{f}(y) y \\
\dot{y}=h\left(g(x) q_{f}(y)\right) q_{r}(y) y-d\left(g(x) q_{f}(y)\right) y .
\end{array}\right.
$$

The latter expression actually encompasses models (1), (2) and (3) when one or both of $q_{f}(y)$ and $q_{r}(y)$ are equal to 1 for all $y$. In this case, the corresponding component Allee effect simply does not occur. Since it is the more general, we will conduct our theoretical investigations on the basis of model (4).

Table 1 synthesizes the assumptions made on the different functions involved in the models. When these functions are specified, e.g. logistic prey growth, Holling II 
functional and numerical responses, constant death rate, the models reduce to classical continuous-time predator-prey models (Rosenzweig and MacArthur 1963) and to their Allee effect variants when $q_{f}(y)$ and/or $q_{r}(y)$ are different from 1 (Bazykin. 1998; Zhou et al, 2005; Verdy, 2010, Terry, 2015).

\subsection{Augmentative biological control}

The representation of a biological control in which predators are periodically released can also be described by hybrid systems (Mailleret and Lemesle, 2009). We also formulate the general predator-prey interactions using systems as (4) and the periodic releases described by a discrete equation.

Augmentative biological control is classically modeled using a periodic discrete time equation to depict predator introductions that imply sudden increases in their population (Lu et al, 2003). The following equation accounts for such a variation (Mailleret and Lemesle, 2009; Mailleret and Grognard, 2009):

$$
y\left(n T^{+}\right)=y(n T)+\mu T, n \in \mathbb{N},
$$

denoting $n T^{+}$the time right after $n T$. In this model, a quantity $\mu T$ of predators is introduced at each time $n T$. Parameter $\mu$ denotes the rate of introduction rate of predators into the targeted domain, i.e. the number of introduced predators per unit time, and $T$ the period of introduction. This model of predator introductions allows to consider a gradient of predator release strategies with a given $\mu$, from small and frequent natural enemies introductions to large but rare ones.

Combining equations (4) and (5), the complete augmentative biological control model reads $\forall n \in \mathbb{N}$ :

$$
\left\{\begin{array}{l}
\dot{x}=f(x)-g(x) q_{f}(y) y, \\
\dot{y}=h\left(g(x) q_{f}(y)\right) q_{r}(y) y-d\left(g(x) q_{f}(y)\right) y, \\
y\left(n T^{+}\right)=y(n T)+\mu T .
\end{array}\right.
$$

\section{Stability of the pest-free solution}

In this section, we study the existence of a $T$-periodic, pest-free, stationary solution for Model (6); the conditions for its local stability are also investigated.

3.1 Existence of a pest-free stationary solution

Proposition 1 Under Hypotheses of Table 1, Model (6) admits a pest-free solution:

$$
\left(x_{p}(t), y_{p}(t)\right)=\left(0, y^{*} e^{-d_{0}(t \bmod T)}\right),
$$

with

$$
y^{*}=\frac{\mu T}{1-e^{-d_{0} T}},
$$

which is a T-periodic solution of system 6 . 
After noticing that when $x=0$, predator dynamics reduce to

$$
\dot{y}=h\left(g(0) q_{f}(y)\right) q_{r}(y) y-d\left(g(0) q_{f}(y)\right) y=-d_{0} y,
$$

Proposition 1 follows from standard arguments, see e.g. (Mailleret and Grognard, 2009).

Note that the solution is the same for all variations of the model because the reasoning does not depend on $q_{f}($.$) and q_{r}($.$) .$

\subsection{Stability of the pest-free solution}

In this section we look for the conditions under which the pest-free solution (7) is stable, i.e. under what conditions predators can efficiently suppress pests.

Theorem 2 Pest-free solution (7) is Locally Asymptotically Stable (LAS) for Model (6) if and only if

$$
\Psi_{L A S}(T, \mu)=\int_{0}^{T} q_{f}\left(y_{p}(\tau)\right) y_{p}(\tau) d \tau-\frac{f^{\prime}(0)}{g^{\prime}(0)} T>0 .
$$

Moreover, if

$$
\Psi_{G A S}(T, \mu)=\int_{0}^{T} q_{f}\left(y_{p}(\tau)\right) y_{p}(\tau) d \tau-S T>0
$$

then solution (7) is Globaly Asymptotically Stable (GAS).

Proof Uniqueness of solution (6) is obvious since all functions are $\mathscr{C}^{1}$ and the jumps are state independent. Hence, non-negativity of solutions is ensured since $\dot{x}=0$ when $x=0, \dot{y}=0$ when $y=0$ and $y\left(n T^{+}\right)>y(n T) \geqslant 0$.

To obtain the global stability conditions of the pest-free solution, both the local stability condition and a sufficient global attractivity condition are computed.

System (4) is first written in deviation coordinates $(\widetilde{x}(t), \widetilde{y}(t))$ which is the difference between a general solution noted $(x(t), y(t))$ and the periodic pest-free solution $\left(x_{p}(t), y_{p}(t)\right)$ :

$$
\left\{\begin{array}{l}
\widetilde{x}(t)=x(t)-x_{p}(t)=x(t) \\
\widetilde{y}(t)=y(t)-y_{p}(t)
\end{array}\right.
$$

System (4) becomes then

$$
\left\{\begin{array}{l}
\dot{\tilde{x}}=f(\widetilde{x})-g(\widetilde{x}) q_{f}\left(y_{p}+\widetilde{y}\right)\left(y_{p}+\widetilde{y}\right), \\
\dot{\tilde{y}}=h\left(g(\widetilde{x}) q_{f}\left(y_{p}+\widetilde{y}\right)\right) q_{r}\left(\widetilde{y}+y_{p}\right)\left(y_{p}+\widetilde{y}\right)-d\left(g(\widetilde{x}) q_{f}\left(y_{p}+\widetilde{y}\right)\right)\left(y_{p}+\widetilde{y}\right)+d_{0} y_{p},
\end{array}\right.
$$

where the impulsive component disappears since $\widetilde{y}\left(n T^{+}\right)=y(n T)+\mu T-y_{p}(n T)-$ $\mu T=\widetilde{y}(n T)$. 
To obtain the attractivity conditions, the convergence of deviation coordinates to zero is first demonstrated. We divide the pest dynamics by $g(\widetilde{x}(t))$ :

$$
\frac{\dot{\tilde{x}}}{g(\widetilde{x})}=\frac{f(\widetilde{x})}{g(\widetilde{x})}-q_{f}\left(y_{p}+\widetilde{y}\right)\left(y_{p}+\widetilde{y}\right),
$$

and we integrate over state and time domains the left- and right-hand terms, respectively:

$$
\int_{\widetilde{x}\left(t_{0}\right)}^{\widetilde{x}(t)} \frac{d \widetilde{x}}{g(\widetilde{x})}=\int_{t_{0}}^{t}\left[\frac{f(\widetilde{x}(\tau))}{g(\widetilde{x}(\tau))}-q_{f}\left(y_{p}(\tau)+\widetilde{y}(\tau)\right)\left(y_{p}(\tau)+\widetilde{y}(\tau)\right)\right] d \tau
$$

Since $\forall \widetilde{x}>0, g(\widetilde{x})>0$, and $g(\widetilde{x}) \approx g^{\prime}(0) x$ with $g^{\prime}(0)>0$ for small values of $\tilde{x}$, the left side of the latter equation goes to $-\infty$ if and only if $\widetilde{x}(t)$ converges to zero (because $\widetilde{x}\left(t_{0}\right)>\widetilde{x}(t)$ in this case). Then, $\widetilde{x}(t)$ converges to zero for $t \rightarrow+\infty$ if and only if the right-hand side of (11) goes to $-\infty$.

Considering now the $\tilde{y}$ dynamics in equation (10), assumptions on function $d($. (Table 1) imply that $-d\left(g(x) q_{f}(y)\right) \geqslant-d_{0}$, so that:

$$
\dot{\tilde{y}} \geqslant h\left(g(\widetilde{x}) q_{f}\left(y_{p}+\widetilde{y}\right)\right) q_{r}\left(y_{p}+\widetilde{y}\right)\left(y_{p}+\widetilde{y}\right)-d_{0} \tilde{y} \geqslant-d_{0} \tilde{y} .
$$

Thus $\forall t \geq 0, \widetilde{y}(t) \geqslant \widetilde{y}_{0} e^{-d_{0}\left(t-t_{0}\right)}$. Moreover, $\widetilde{y}(t) \geqslant-y_{p}(t)$ because $y=\widetilde{y}+y_{p}$ remains non-negative. Thus $\widetilde{y} \geqslant-\max y_{p}(t)=-y^{*}$, so that $\widetilde{y_{0}} \geqslant-y^{*}$ and:

$$
\widetilde{y}(t) \geqslant-y^{*} e^{-d_{0}\left(t-t_{0}\right)} .
$$

Thus, $\forall \varepsilon>0$ there exists a time $t_{f}$ such that $\forall t>t_{f}$ :

$$
\widetilde{y}(t)>-\varepsilon \text {. }
$$

For $t$ large enough, we split the right hand side of equation (11) in three integrals of the same integrand 1

$$
\left(\int_{t_{0}}^{\left(\left\lfloor\frac{t_{f}}{T}\right\rfloor+1\right) T}+\int_{\left(\left\lfloor\frac{t_{f}}{T}\right\rfloor+1\right) T}^{\left\lfloor\frac{t}{T}\right\rfloor T}+\int_{\left\lfloor\frac{t}{T}\right\rfloor T}^{t}\right)\left[\frac{f(\widetilde{x}(\tau))}{g(\widetilde{x}(\tau))}-q_{f}\left(y_{p}(\tau)+\widetilde{y}(\tau)\right)\left(y_{p}(\tau)+\widetilde{y}(\tau)\right)\right] d \tau .
$$

In this equation, the sum of the first and third integral are upper bounded since $\left[t_{0},\left(\left\lfloor\frac{t_{f}}{T}\right\rfloor+1\right) T\right]$ and $\left[\left\lfloor\frac{t}{T}\right\rfloor T, t\right]$ have finite length and $f(.) / g($.$) is upper bounded (Table$ 1).

1 The following notation has been used to split an integral into 3 integrals:

$$
\int_{t_{0}}^{t} F(\tau) d \tau=\int_{t_{0}}^{t_{1}} F(\tau) d \tau+\int_{t_{1}}^{t_{2}} F(\tau) d \tau+\int_{t_{2}}^{t} F(\tau) d \tau=\left(\int_{t_{0}}^{t_{1}}+\int_{t_{1}}^{t_{2}}+\int_{t_{2}}^{t}\right) F(\tau) d \tau
$$


The second integral is such that:

$$
\begin{aligned}
\int_{\left(\left\lfloor\frac{t_{f}}{T}\right\rfloor+1\right) T}^{\left\lfloor\frac{t}{T}\right\rfloor T}\left[\frac{f(\widetilde{x}(\tau))}{g(\widetilde{x}(\tau))}-q_{f}\left(y_{p}(\tau)+\widetilde{y}(\tau)\right)\left(y_{p}(\tau)+\widetilde{y}(\tau)\right)\right] d \tau \\
\leqslant \int_{\left(\left\lfloor\frac{t_{f}}{T}\right\rfloor+1\right) T}^{\left\lfloor\frac{t}{T}\right\rfloor T}\left[S-q_{f}\left(y_{p}(\tau)+\widetilde{y}(\tau)\right)\left(y_{p}(\tau)+\widetilde{y}(\tau)\right)\right] d \tau, \\
\leqslant \sum_{n=\left\lfloor\frac{t_{f}}{T}\right\rfloor+1}^{\left\lfloor\frac{t}{T}\right\rfloor-1} \int_{n T}^{(n+1) T}\left[S-q_{f}\left(y_{p}(\tau)-\varepsilon\right)\left(y_{p}(\tau)-\varepsilon\right)\right] d \tau \\
=\left(\left\lfloor\frac{t}{T}\right\rfloor-\left\lfloor\frac{t_{f}}{T}\right\rfloor-1\right) \int_{0}^{T}\left[S-q_{f}\left(y_{p}(\tau)-\varepsilon\right)\left(y_{p}(\tau)-\varepsilon\right)\right] d \tau .
\end{aligned}
$$

The first inequation comes from the upper boundedness of $f(.) / g($.$) . The second is$ due to 13 combined with the fact that $q_{f}(y) y$ is increasing in $y$ (Table 1). The third equality stems from the $T$-periodicity of $y_{p}(t)$ (Proposition 1 ).

The term $\left(\left\lfloor\frac{t}{T}\right\rfloor-\left\lfloor\frac{t_{f}}{T}\right\rfloor-1\right)$ is positive and goes to $+\infty$. If it is multiplied by some negative constant, the right-hand term of (11) goes to $-\infty$ as $t \rightarrow \infty$. Thus $\widetilde{x}(t)$ converges to zero if there exists $\varepsilon>0$ such that:

$$
S T-\int_{0}^{T} q_{f}\left(y_{p}(\tau)-\varepsilon\right)\left(y_{p}(\tau)-\varepsilon\right) d \tau<0
$$

The latter equation, is a small perturbation of (9). Thus, provided condition (9) holds, there exists $\varepsilon>0$ such that (14) is satisfied and $x(t)$ asymptotically converges to $x_{p}(t)=0$. Therefore 99 is a sufficient condition for the convergence of $x(t)$ to 0 .

Regarding the convergence of $y(t)$ to $y_{p}(t)$ when (9) holds, we first come back to the second equation of 10 . The case where $\widetilde{y}(0) \geqslant 0$ is first considered. Since $\dot{\tilde{y}}(\tilde{y}=0) \geqslant 0, \widetilde{y}$ can not take negative values. Since $(9)$ holds, $\widetilde{x}$ converges to zero with time, then $h\left(g(\widetilde{x}) q_{f}\left(y_{p}+\widetilde{y}\right)\right)$, which is smaller than $h(g(\widetilde{x}))$, tends to zero too. By the same token, $d\left(g(\widetilde{x}) q_{f}\left(y_{p}+\widetilde{y}\right)\right)$ converges to $d_{0}$ : it means that $d\left(g(\widetilde{x}) q_{f}\left(y_{p}+\widetilde{y}\right)\right)$ becomes larger or equal to $d_{0} / 2$ for $t$ sufficiently large since $d($.$) is non increasing.$ Thus,

$$
\begin{aligned}
\dot{\tilde{y}} & =\left[h\left(g(\widetilde{x}) q_{f}\left(y_{p}+\widetilde{y}\right)\right) q_{r}\left(y_{p}+\widetilde{y}\right)-d\left(g(\widetilde{x}) q_{f}\left(y_{p}+\widetilde{y}\right)\right)+d_{0}\right] y_{p} \\
& +\left[h\left(g(\widetilde{x}) q_{f}\left(y_{p}+\widetilde{y}\right)\right) q_{r}\left(y_{p}+\widetilde{y}\right)-d\left(g(\widetilde{x}) q_{f}\left(y_{p}+\widetilde{y}\right)\right)\right] \widetilde{y}, \\
& \leqslant\left[h\left(g(\widetilde{x}) q_{f}\left(y_{p}+\widetilde{y}\right)\right) q_{r}\left(y_{p}+\widetilde{y}\right)-d\left(g(\widetilde{x}) q_{f}\left(y_{p}+\widetilde{y}\right)\right)+d_{0}\right] y_{p}-\frac{d_{0}}{2} \widetilde{y} .
\end{aligned}
$$

and $\tilde{y}$ goes to zero for large values of $t$ when (9) holds since the first term of the right-hand side goes to zero and $\widetilde{y}$ is non negative.

For a negative value of $\widetilde{y}(0)$, either $\tilde{y}$ becomes positive at some $t>0$ and the previous reasoning applies, or it does not and equation 12 proves that $\tilde{y}$ converges 
to zero. Thus we have proved that the pest-free solution is globally attractive under condition (9).

Concerning the local stability of solution (7), system (10) is first linearized around the pest-free solution (Khalil and Grizzle, 2002). This yields in matrix form:

$$
\left(\begin{array}{c}
\dot{\tilde{x}} \\
\dot{\tilde{y}}
\end{array}\right)=\left(\begin{array}{cc}
f^{\prime}(0)-g^{\prime}(0) q_{f}\left(y_{p}\right) y_{p} & 0 \\
\left.\left(h^{\prime}(0) q_{f}\left(y_{p}\right)-d^{\prime}(0)\right) g^{\prime}(0)\right) q_{f}\left(y_{p}\right) y_{p} & -d_{0}
\end{array}\right)\left(\begin{array}{l}
\tilde{x} \\
\widetilde{y}
\end{array}\right) .
$$

This allows to compute the post-release mapping of the linearized model

$$
\left(\begin{array}{c}
\tilde{x} \\
\tilde{y}
\end{array}\right)\left((n+1) T^{+}\right)=B\left(\begin{array}{c}
\tilde{x} \\
\tilde{y}
\end{array}\right)\left(n T^{+}\right)
$$

with $B$ the monodromy matrix (Floquet, 1883; Lakshmikantham et al, 1989) such that

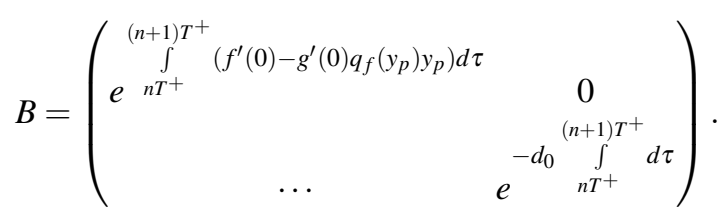

The matrix being lower triangular, the bottom-left term does not influence the local stability analysis and is left unspecified. The Floquet's characteristic multipliers are the eigenvalues of this matrix and to obtain the local stability of the pest-free solution, these terms must be smaller than 1 (Floquet, 1883). Note that since the two integrands are $T$-periodic because $y_{p}(\tau)$ is, we can then set $n=0$. The second eigenvalue being always smaller than 1 , local stability condition of the pest free solution is achieved if and only if the following inequality holds:

$$
\frac{f^{\prime}(0)}{g^{\prime}(0)} T-\int_{0}^{T^{+}} q_{f}\left(y_{p}(\tau)\right) y_{p}(\tau) d \tau<0,
$$

which is actually (8). The difference between the latter inequality and condition (9) lies in the terms $S=\sup _{x \geqslant 0}\left(\frac{f(x)}{g(x)}\right)$ and $\frac{f^{\prime}(0)}{g^{\prime}(0)}$. However, $\lim _{x \rightarrow 0} \frac{f(x)}{g(x)}=\frac{f^{\prime}(0)}{g^{\prime}(0)}$ due to l'Hospital rule so that $\frac{f^{\prime}(0)}{g^{\prime}(0)} \leqslant S$. The global attractivity condition is stronger than the local stability condition so that it is the global stability condition for the pest-free solution.

Note that Theorem 2, built on model (6) can be used with models derived from (2), (3) and (1) by setting $q_{f}$ and/or $q_{r}$ to the constant function $q_{i}(y)=1$, for $i=f$ and / or $r$. For model 2,, where only foraging efficiency is affected, it is obvious that we obtain exactly the same conditions for both local and global stability of the pest-free solution as for (6), since only $q_{f}$ (and not $q_{r}$ ) impacts conditions (8) and (9). Concerning Models without the foraging efficiency component Allee effect (3) and (1), conditions (8) and (9) simplify, respectively into:

$$
\mu>d_{0} \frac{f^{\prime}(0)}{g^{\prime}(0)},
$$


and

$$
\mu>d_{0} S \text {. }
$$

Note that (18) and (19) where already given in (Mailleret and Grognard, 2009) for models without Allee effects. We show that they are also valid for the reproductive component Allee effect models. It is worth noticing that conditions (18) and (19) are independent on the release period $T$, contrary to (8) and (9). They only depend on the introduction rate $\mu$ which has to be large enough to obtain stability. These results are summarized in Table 3

Since the predators can not disappear due to their periodic releases, pest extinction is actually what is of interest for stability. The pest loss depends directly on how efficient the predation is, which is greatly influenced by the foraging Allee effects. Actually, LAS condition (8) can be seen as a balance condition for the pest class in the vicinity of the pest-free solution over the $T$-time interval, as deduced from (15). GAS condition (97) states the fact that cumulated pest loss due to predation exceeds pest growth over the $T$-time interval anywhere in the domain, as can be seen from the link between (11) and (14) (Zhang et al, 2008; Terry, 2014b).

GAS condition (8) leads the pest population to eradication by forcing the pests to be fewer after each introduction, say $x\left((n+1) T^{+}\right)<x\left(n T^{+}\right)$, for $n \in \mathbb{N}$. Another intuitive condition could be that the pest population always decreases when it interacts with predators, that is $\dot{x}<0$, after some time. Such a property is investigated in what follows.

Corollary 1 If the condition

$$
q_{f}\left(y_{p}(T)\right) y_{p}(T)>S
$$

holds, then the pest-free solution is GAS and there exists a time $t_{c}>0$ after which $\dot{x}<0$ for all $t>t_{c}$.

Moreover, if $y(0)>y_{p}(0)$, then $t_{c}=0$.

Proof Since the function $q_{f}(y) y$ is non-decreasing and $y_{p}(t)$ is decreasing over $(0, T]$, it is clear that

$$
\int_{0}^{T} q_{f}\left(y_{p}(\tau)\right) y_{p}(\tau) d \tau>q_{f}\left(y_{p}(T)\right) y_{p}(T) T .
$$

Hence, condition (9) will hold provided that $q_{f}\left(y_{p}(T)\right) y_{p}(T) T>S T$, which is equivalent to (20). Condition 20] then ensures GAS.

We now investigate the sign of the pest dynamics which can be upper-bounded as follows:

$$
\dot{x} \leqslant g(x)\left(S-q_{f}(y) y\right) .
$$

Also, since $y(t)$ converges to $y_{p}(t), q_{f}(y(t)) y(t)$ converges to $q_{f}\left(y_{p}(t)\right) y_{p}(t)$. Hence, for all $\varepsilon>0$, there exists a time $t_{c}$ such that for all times $t>t_{c}$ :

$$
q_{f}(y(t)) y(t)+\varepsilon>q_{f}\left(y_{p}(t)\right) y_{p}(t) .
$$

By choosing $\varepsilon=q_{f}\left(y_{p}(T)\right) y_{p}(T)-S>0$, we have that

$$
q_{f}(y(t)) y(t)+q_{f}\left(y_{p}(T)\right) y_{p}(T)-S>q_{f}\left(y_{p}(t)\right) y_{p}(t),
$$


after the corresponding $t_{c}$, leading to

$$
S-q_{f}(y(t)) y(t)<q_{f}\left(y_{p}(T)\right) y_{p}(T)-q_{f}\left(y_{p}(t)\right) y_{p}(t) \leqslant 0,
$$

for all $t>t_{c}$ because $q_{f}(y) y$ is non-decreasing and $y_{p}(t)$ has its minimum in $T$. Thus, from $(21)$, we have that

$$
\dot{x}<g(x)\left(q_{f}\left(y_{p}(T)\right) y_{p}(T)-q_{f}\left(y_{p}(t)\right) y_{p}(t)\right)<0 .
$$

Moreover, using the reasoning from the proof of Theorem 2 saying that $\tilde{y}$ stays non-negative if its initial value $\tilde{y}(0)$ is non-negative (After Equation (14)), we know that if $y(0) \geqslant y_{p}(0), y(t) \geqslant y_{p}(t)$ for all times $t \geqslant 0$. This leads directly to the satisfaction of (22) for all times and all $\varepsilon>0$. Hence, $\dot{x}<0$ for all $t \geqslant 0$.

Condition (20) can be written as

$$
\mu>\frac{e^{d_{0} T}-1}{T}\left(q_{f}(y) y\right)^{-1}(S)
$$

in which $\left(q_{f}(y) y\right)^{-1}(\cdot)$ is the inverse function of $q_{f}(y) y$. The threshold value of $\mu$, the right-hand side, is then increasing with respect to the period $T$. The increase of the threshold value of $\mu$ of condition 24 with $T$ can be explained because restrictions to obtain condition 20) are stringent. Indeed, even the smallest predator density $y_{p}(T)$ has to ensure prey decrease $\dot{x}<0$. Since $y_{p}(T)$ tends to become very small for large $T$ values, large $\mu$ values are necessary to counter this effect. On the contrary, no monotonicity feature was found in (9) due to the genericity of the functions involved within the integrals (but see Section 4.2 for such an analysis in a specific case).

In what follows, we investigate deeper the domain for which we have local stability and no global stability. Although the reproductive component Allee effect does not impact the stability conditions, results in the next section reveals how such a mechanism can affect the Non Global Stability, which is defined in the next part.

\subsection{When the pest-free solution is LAS but not GAS}

When $S=\frac{f^{\prime}(0)}{g^{\prime}(0)}$, LAS and GAS conditions are equivalent. Otherwise there can be situations where the pest-free solution is LAS and not GAS (Terry, 2014a). In such situations, we will evidence that the reproductive component Allee effect does have an impact on the global attractivity of the pest-free solution. This was not apparent in conditions (8) and (9).

To go further, we had to assume the quite natural hypothesis that the predator functional response is upper bounded by some $g_{m}>0$ when time is large enough. This can directly come from the boundedness of the functional response itself (first line of hypothesis in Table 2), which is most of the time the case except for linearlike functional responses. Otherwise, this can also stem from a logistic-like growth of the prey, imposing negative prey growth for large prey density, which imply that when the time is large enough, prey density is upper bounded, and so is the functional 


\begin{tabular}{ll}
\hline Function & Hypothesis \\
\hline$g(x)$ & $\exists g_{m}>0$ such that $g(x) \leqslant g_{m}$ for all $x \geqslant 0$ \\
$f(x)$ & $\exists K$ such that $f(x)<0$ for $x>K$ \\
\hline
\end{tabular}

Table 2 Additional hypotheses either on function $f($.$) or on function g($.$) for the non global stability study.$ Both hypotheses imply that the predator functional response is upper bounded by some $g_{m}>0$ when time is large enough.

response (second line of hypothesis in Table 2). Without loss of generality, we will suppose that $g(x(t))<g_{m}, \forall t \geq 0$ in what follows.

When the pest-free solution is LAS but not GAS, we refer to Non Global Stability (NGS). The following technical lemma defines a function which exists when LAS condition (8) is satisfied:

Lemma 3 There exists a function $\Phi($.$) such that$

$$
\Psi_{L A S}(T, \mu)>0 \Leftrightarrow \mu>\Phi(T)
$$

Proof When $t$ is fixed, $y_{p}(t)$ is strictly increasing in $\mu$; so is $q_{f}\left(y_{p}(t)\right) y_{p}(t)$ so that $\Psi_{L A S}(T, \mu)$ is also strictly increasing in $\mu . \Psi_{L A S}($.$) is a continuously differentiable$ function on $\mathbb{R}_{+}^{*} \times \mathbb{R}_{+}$, and its derivative with respect to $\mu$ is strictly positive. Using the implicit function theorem one can show that there exists a continuously differentiable function $\Phi($.$) such that:$

$$
\Psi_{L A S}(T, \mu)=0 \Leftrightarrow \Phi(T)=\mu
$$

As $\Psi_{L A S}($.$) is increasing with \mu$, the LAS condition of the pest-free solution (8) can be rewritten as:

$$
\mu>\Phi(T)
$$

The NGS condition is then:

Theorem 4 If $d\left(g_{m}\right)-h\left(g_{m}\right)>0$ and function $\Phi($.$) defined in Lemma 3$ satisfies

$$
\Phi(T)<\frac{1}{T} \inf _{y>y_{S}}\left(y\left(1-e^{-\theta(y) T}\right)\right)
$$

with $y_{S}$ such that $q_{f}\left(y_{S}\right) y_{S}=S$, and

$$
\theta(y)=d\left(g_{m} q_{f}(y)\right)-h\left(g_{m} q_{f}(y)\right) q_{r}(y),
$$

then there exist values of $\mu$ such that pest-free solution is LAS but not GAS. 
Proof Under the assumption that the pest-free solution is LAS, the proof is based on the idea of finding sufficient parametric conditions such that there exists a prey level $x=x_{c} \leqslant x(0)$ implying $\dot{x} \geqslant 0$ for all possible values of $y$. This would then prevent convergence of $x$ to zero for initial conditions with $x(0) \geqslant x_{c}$.

Suppose first that the predator population is upper bounded over time by a positive constant $y_{m}$ (this bound will be explicited later in the proof). The pest dynamics would be lower bounded by:

$$
\dot{x} \geqslant g(x)\left(\frac{f(x)}{g(x)}-q_{f}\left(y_{m}\right) y_{m}\right),
$$

so that from here, it would be sufficient to have

$$
S \geqslant q_{f}\left(y_{m}\right) y_{m},
$$

to ensure that there would exist $x_{c}$ such that $\frac{f\left(x_{c}\right)}{g\left(x_{c}\right)}>q_{f}\left(y_{m}\right) y_{m}$. As $q_{f}(y) y$ is increasing in $y$, the smaller $y_{m}$ is, the greater the set of $x$ satisfying $\dot{x} \geqslant 0$ would be. The key of the proof is thus to find such (small) $y_{m}$.

Arguably, the smallest $y_{m}$ can be found from the trajectories initiated with the smallest predator population density: $y(0)=0$. The computation of $y_{m}$ is based on a recursive definition of a sequence $\left(y_{m_{i}}\right)_{i \in \mathbb{N}}$ of upper bounds of $y(t)$ initiated at $y(0)=$ 0 . In absence of any further information, the sequence is initiated at $y_{m_{0}}=+\infty$.

Assume that $y(t)<y_{m_{i}}$ for some $i \in \mathbb{N}$ and that $d\left(g_{m}\right)-h\left(g_{m}\right)>0$. Using the hypotheses on the variation of functions $q_{i}(),. h($.$) and d($.$) (Table 1 p$ together with the boundedness of $g($.$) (Table 2), one gets that$

$$
\dot{y} \leqslant-\theta\left(y_{m_{i}}\right) y,
$$

with $\theta\left(y_{m_{i}}\right)=d\left(g_{m} q_{f}\left(y_{m_{i}}\right)\right)-h\left(g_{m} q_{f}\left(y_{m_{i}}\right)\right) q_{r}\left(y_{m_{i}}\right) \geqslant d\left(g_{m}\right)-h\left(g_{m}\right)>0$, because $q_{f}($.$) and q_{r}($.$) are smaller than 1$.

Inequation (30) is a linear autonomous differential inequation in dimension 1. Classical results on impulsive differential inequations (Lakshmikantham et al, 1989) imply that the trajectory $y(t)$ of the original model with $y(0)=0$ is upper-bounded by the solution of the following system

$$
\left\{\begin{array}{l}
\dot{y_{i}}=-\theta\left(y_{m_{i}}\right) y_{i}, \text { for } t \neq n T, \\
y_{i}\left(n T^{+}\right)=y_{i}(n T)+\mu T, \\
y_{i}(0)=y(0)=0 .
\end{array}\right.
$$

The solution is such that $y_{i}\left((n+1) T^{+}\right)=y_{i}\left(n T^{+}\right) e^{-\theta\left(y_{m_{i}}\right) T}+\mu T$. The sequence $\left(y_{i}\left(n T^{+}\right)\right)_{n \in \mathbb{N}}$ is non-decreasing from $y_{i}\left(0^{+}\right)=y(0)+\mu T$. Since $\theta\left(y_{m_{i}}\right)>0$, the sequence $\left(y_{i}\left(n T^{+}\right)\right)_{n \in \mathbb{N}}$ converges to the fixed point

$$
\frac{\mu T}{1-e^{-\theta\left(y_{m_{i}}\right) T}}=W\left(y_{m_{i}}\right) .
$$


Since $y_{i}(t) \leqslant y_{i}\left(n T^{+}\right), \forall t \in\left(n T^{+},(n+1) T\right]$ and $\forall n \in \mathbb{N}$, it is clear that $y_{i}(t) \leqslant W\left(y_{m_{i}}\right)$, $\forall t \geqslant 0$. Thus $W\left(y_{m_{i}}\right)$ is also an upper bound of $y(t)$ initiated at $y(0)=0$, and we can use it as a basis for the definition of the sequence $\left(y_{m_{i}}\right)_{i \in \mathbb{N}}$ :

$$
y_{m_{i+1}}=W\left(y_{m_{i}}\right) \text {. }
$$

The remaining part of the proof relies on the study of the sequence defined by 32 in order to obtain the best possible upper bound for $y(t)$.

One first notes that $W(0)=\frac{\mu T}{1-e^{-\theta(0) T}}>0$, and that $\lim _{z \rightarrow+\infty} W(z)=W\left(y_{m_{0}}\right)=y_{m_{1}}$ is finite because $\lim _{z \rightarrow+\infty} \theta(z)=\theta\left(y_{m_{0}}\right)=d\left(g_{m}\right)-h\left(g_{m}\right)>0$. Thus $W($.$) must have at$ least one fixed point between 0 and $+\infty$. These fixed points are necessarily smaller than $y_{m_{1}}$ since the latter is the supremum of $W($.$) on \mathbb{R}^{+}$.

Let us denote $y_{m}^{*}$ the largest fixed point. Then $\forall z \in\left(y_{m}^{*},+\infty\right), W(z) \in\left(y_{m}^{*}, y_{m_{1}}\right)$, so that, $W($.$) being non-decreasing, any sequence (32) initiated above y_{m}^{*}$ monotonically converge to $y_{m}^{*}$. In particular this is the case of the sequence considered here since $y_{m_{0}}=+\infty$ and $y_{m}^{*}$ is the infimum of the sequence. Thus, $y_{m}^{*}$ is the smallest upper bound of $y(t)$ that can be obtained from (32).

For initial conditions to exist such that the corresponding trajectory do not converge to the pest free solution, i.e. that the pest free solution is not GAS, it then suffices that 29] holds for $y_{m}=y_{m}^{*} \cdot y_{m}^{*}$ is defined as the largest solution of $y=W(y)$ and one has the property that for all $y>y_{m}^{*}, W(y)<y$.

Remembering that $q_{f}(y) y$ is increasing in $y$ we define $y_{S}$ such that $q_{f}\left(y_{S}\right) y_{S}=S$. Then, if

$$
\forall y>y_{S}, W(y)<y,
$$

one can conclude that $y_{S}$ is larger or equal to $y_{m}^{*}$, i.e. that 29) holds with $y=y_{m}^{*}$. From the definition of $W($.$) , condition 33$ is equivalent to have $\mu<\frac{1}{T}\left(1-e^{-\theta(y) T}\right) y$ for all $y>y_{S}$, so that a NGS condition is:

$$
\mu<\frac{1}{T} \inf _{y>y_{S}}\left(\left(1-e^{-\theta(y) T}\right) y\right) .
$$

Combining this with the LAS condition (8), obtained in Lemma 3 , the pest-free solution is LAS and not GAS if

$$
\Phi(T)<\mu<\frac{1}{T} \inf _{y>y_{S}}\left(\left(1-e^{-\theta(y) T}\right) y\right)
$$

which concludes the proof.

The condition $d\left(g_{m}\right)-h\left(g_{m}\right)>0$ can be interpreted as: in absence of releases, the predator class is decreasing even if the population of prey is at its maximum level, which is the most favorable situation for predator growth. This first consideration then leads to the pest increase at all times. In other words, keeping the predator population from becoming too large prevents the prey population from becoming small.

The NGS condition (27) is actually easier to satisfy when the predator population is subject one or the other component Allee effect, and even more whent it is to both of them. Indeed, because $q_{f}($.$) and q_{r}($.$) are non-decreasing and between 0$ and 1 , and that $d($.$) and h($.$) are respectively non-increasing and non-decreasing, \theta(y)$ is larger 


\begin{tabular}{lll}
\hline Model & Stability & Condition \\
\hline \multirow{2}{*}{$\begin{array}{l}\text { Foraging and double } \\
\text { Allee effect }\end{array}$} & LAS & $\frac{g^{\prime}(0)}{T} \int_{0}^{T} q_{f}\left(y_{p}(\tau)\right) y_{p}(\tau) d \tau-f^{\prime}(0)>0$ \\
\cline { 2 - 3 } & GAS & $\frac{g^{\prime}(0)}{T} \int_{0}^{T} q_{f}\left(y_{p}(\tau)\right) y_{p}(\tau) d \tau-S g^{\prime}(0)>0$ \\
\cline { 2 - 3 } & NGS & $\begin{array}{l}\frac{1}{T} \inf _{y>y_{S}}\left(\left(1-e^{-\theta(y) T}\right) y\right)-\mu<0 \\
\theta(y)=d\left(g_{m} q_{f}(y)-h\left(g_{m} q_{f}(y) q_{r}(y)\right.\right.\end{array}$ \\
\hline \multirow{2}{*}{$\begin{array}{l}\text { Reproductive Allee } \\
\text { effect }\end{array}$} & LAS & $\mu-d_{0} \frac{f^{\prime}(0)}{g^{\prime}(0)}>0$ \\
\cline { 2 - 3 } & GAS & $\mu-d_{0} S>0$ \\
\cline { 2 - 3 } & NGS & $\begin{array}{l}\frac{1}{T} \inf _{y>y_{S}}\left(\left(1-e^{-\theta(y) T}\right) y\right)-\mu<0 \\
\theta(y)=d\left(g_{m}\right)-h\left(g_{m}\right) q_{r}(y)\end{array}$ \\
\hline
\end{tabular}

Table 3 Summary of stability conditions for models 6 and this derived from 3 . Models derived from (2) and (1) are not presented here since stability conditions are similar to those from models (6) and (3): the function $q_{r}$ is then equal to 1 in NGS conditions. Note also that LAS condition needs to be satisfied for NGS conditions.

when Allee effects come into play. Therefore, the right hand side of 27] is larger, making more room for finding $\mu$ satisfying [34), i.e. for the pest free solution to be LAS but not GAS.

A direct consequence is also that, in contrast with the local and global stability conditions, the NGS condition (27) is influenced by the reproductive Allee effect. The model is more prone to having the pest free solution LAS but not GAS when the predator population is subject to such mechanism.

\section{Numerical simulations}

In this section, we illustrate the results obtained in Section 3 . We first introduce direct analytical expressions inspired by literature for prey growth, functional and numerical responses, predator mortality and foraging and reproductive Allee effects. We then evaluate the stability conditions for these specific models and compare the different conditions for a given parameter set. Finally we show some simulations of the efficacy of pest suppression in different scenarios.

\subsection{Model examples}

To illustrate inter- and intra- specific functions involved in the model, we first provide a model in which both component Allee effects affect the predator population, as in (4):

$$
\left\{\begin{array}{l}
\dot{x}=r x\left(1-\frac{x}{K}\right)-\left(\frac{\frac{b_{0}}{a} x}{x+x_{h}}\right)\left(\frac{y}{y+\alpha_{f}}\right) y, \\
\dot{y}=\left(\frac{b_{0} x}{x+x_{h}}\right)\left(\frac{y}{y+\alpha_{f}}\right)\left(\frac{y}{y+\alpha_{r}}\right) y-d_{0} y .
\end{array}\right.
$$


With these expressions, pest growth $f(x)$ is logistic with $r$, the maximum growth rate and $K$, the carrying capacity. The function $g(x)$ is of Holling type II (Holling, 1959): $g(x)=\frac{b_{0}}{a} x /\left(x+x_{h}\right)$ with $b_{0}$ the predator uptake efficiency, $a$ the assimilation efficiency and $x_{h}$ the half-saturation prey density. The captured-prey dependent numerical response is assumed linear such that $h\left(g(x) q_{f}(y)\right)=a g(x) q_{f}(y)$. The predator mortality rate is assumed constant: $d()=.d_{0}$. Functions describing Allee effects are defined following (Zhou et al, 2005; Bazykin, 1998; Verdy, 2010):

$$
q_{f}(y)=\frac{y}{y+\alpha_{f}}
$$

and

$$
q_{r}(y)=\frac{y}{y+\alpha_{r}}
$$

in which parameters $\alpha_{f}$ and $\alpha_{r}$ represent, respectively, the strength of foraging efficiency and reproductive Allee effects.

When $\alpha_{f}$ and/or $\alpha_{r}$ are equal to 0 , the corresponding component Allee effect vanishes and one gets instances of the other models (2), (3) and (1). When $\alpha_{r}=0$ and $\alpha_{f}>0$, then predators are only subject to the foraging efficiency Allee effect. Mathematically, (35) reduces to the first model studied in (Verdy, 2010) (equations (33) and (34) in this article). If $\alpha_{r}>0$ and $\alpha_{f}=0$, then only a reproductive Allee effect occurs in the predator population. This model is the second one studied in (Verdy 2010) (equations (33) and (36)); it was also previously considered in (Bazykin, 1998) and (Zhou et al, 2005). No Allee effect occurs when $\alpha_{r}=0$ and $\alpha_{f}=0$ : this refers to the Rosenzweig-MacArthur model (Rosenzweig and MacArthur, 1963).

The dynamics of the Rosenzweig MacArthur model and of the two single-Allee effects models without impulses are well known (Bazykin, 1998; Zhou et al, 2005, Verdy, 2010; Rosenzweig and MacArthur, 1963). Model (35) incorporating foraging and reproductive component Allee effects is, to our knowledge, new. Yet, a rapid study of its equilibria and their stability shows that it appears to have a behavior qualitatively similar to the foraging Allee effect only model.

In what follows, we will refer to the impulsive model with only foraging efficiency Allee effect as the F-model, the one with only reproductive Allee effect as the R-model and the one without Allee effect as the RMA-model. Model (35) will be referred to as the RF-model.

\subsection{Stability conditions}

Stability conditions obtained in Theorem 2 have the following form for the RF-model:

$$
\begin{gathered}
\Psi_{L A S}(T, \mu)=\frac{\mu}{d_{0}}-\frac{a r x_{h}}{b_{0}}+\frac{\alpha_{f}}{d_{0} T} \log \left(\frac{y^{*} e^{-d_{0} T}+\alpha_{f}}{y^{*}+\alpha_{f}}\right)>0, \\
\Psi_{G A S}(T, \mu)=\frac{\mu}{d_{0}}-S+\frac{\alpha_{f}}{d_{0} T} \log \left(\frac{y^{*} e^{-d_{0} T}+\alpha_{f}}{y^{*}+\alpha_{f}}\right)>0,
\end{gathered}
$$


where it should be noted that $y^{*}$ depends on $\mu$ as seen in Proposition 1 , so that $\mu$ can not easily be isolated. Note that, for both conditions to differ, and hence have a rich behavior to illustrate, it is necessary that $S>\frac{a r x_{h}}{b_{0}}$. This requires $K>x_{h}$ and gives $S=\frac{a r}{4 b_{0} K}\left(K+x_{h}\right)^{2}$.

Condition (20) obtained in Corollary 1 is

$$
\mu>\left(\frac{S+\sqrt{S^{2}+4 \alpha_{f} S}}{2}\right)\left(\frac{e^{d_{0} T}-1}{T}\right) \frac{\mu T}{e^{d_{0} T}-1}-\frac{S+\sqrt{S^{2}+4 \alpha_{f} S}}{2}>0,
$$

for positive values of $T$.

We fix parameter values to: $r=7, K=5.5, b_{0}=1.8, a=0.2, x_{h}=0.6, m=3$, $\alpha_{f}=0$ or 10 and $\alpha_{r}=0$ or 10 . These values will be used for all figures.

Using these parameters, $y\left(1-e^{-\theta(y) T}\right)$ is increasing in $y$, then its infimum in (27) for $y>y_{S}$ is achieved for $y=y_{S}$ with

$$
\begin{aligned}
& \theta\left(y_{S}\right)=m-\frac{b_{0} K}{K+x_{h}} \frac{y_{S}}{y_{S}+\alpha_{f}} \frac{y_{S}}{y_{S}+\alpha_{r}}, \\
& y_{S}=\frac{S+\sqrt{S^{2}+4 S \alpha_{f}}}{2},
\end{aligned}
$$

Remember that stability conditions for the other models can be obtained by setting parameters $\alpha_{f}, \alpha_{r}$ or both to zero ( $y_{S}$ is equal to $S$ when $\alpha_{f}=0$ ).

To illustrate the differences between the models, we first computed the areas of the $(T, \mu)$ plane where the different stability conditions hold (Figure 1). We do not plot the GAS conditions (38) since it does not bring new information: we know from (20) that the threshold value of $\mu$ is increasing with respect to $T$ while GAS condition (37) obtained from Theorem 2 is non-increasing in $T$ for all the four models investigated in this article (see Figure 1). Moreover, this threshold limit when $T$ goes to zero is the same as the the limit of $\Psi_{G A S}(T, \mu)$ when $T$ goes to zero. In fact, the GAS condition (9) is obtained by making really the less conservative assumptions on model functions while forcing pest decrease at any time (20) imposes very stringent restrictions.

Figure 1. (a) shows the differences in the stability conditions between the RF- and F-models: analytical conditions for local and global stability are the same since only $\alpha_{f}$, influences the LAS (LAS curve) and GAS (GAS curve) conditions (2) and $\alpha_{r}$, the only difference between the two models, does not come into play in these conditions. We also represented the NGS conditions which are influenced by $\alpha_{r}$ (RF- and F-NGS curves). In addition, because the GAS conditions are only sufficient conditions, we computed their numerical version which are likely more stringent (RF- and F-nGAS curves). The nGAS curves where built by running the corresponding model in Matlab (MATLAB, 2012) with $[K, 0]$ as initial condition, which is deemed to be the initial condition for which pest eradication is the hardest to achieve. We will consider that, when convergence to the pest-free solution occurs, GAS is obtained (numerically, we check for convergence at time $t=3000$ ). Figure 1(b) makes the same type of comparisons between the R-model and the RMA-model. 
(a)
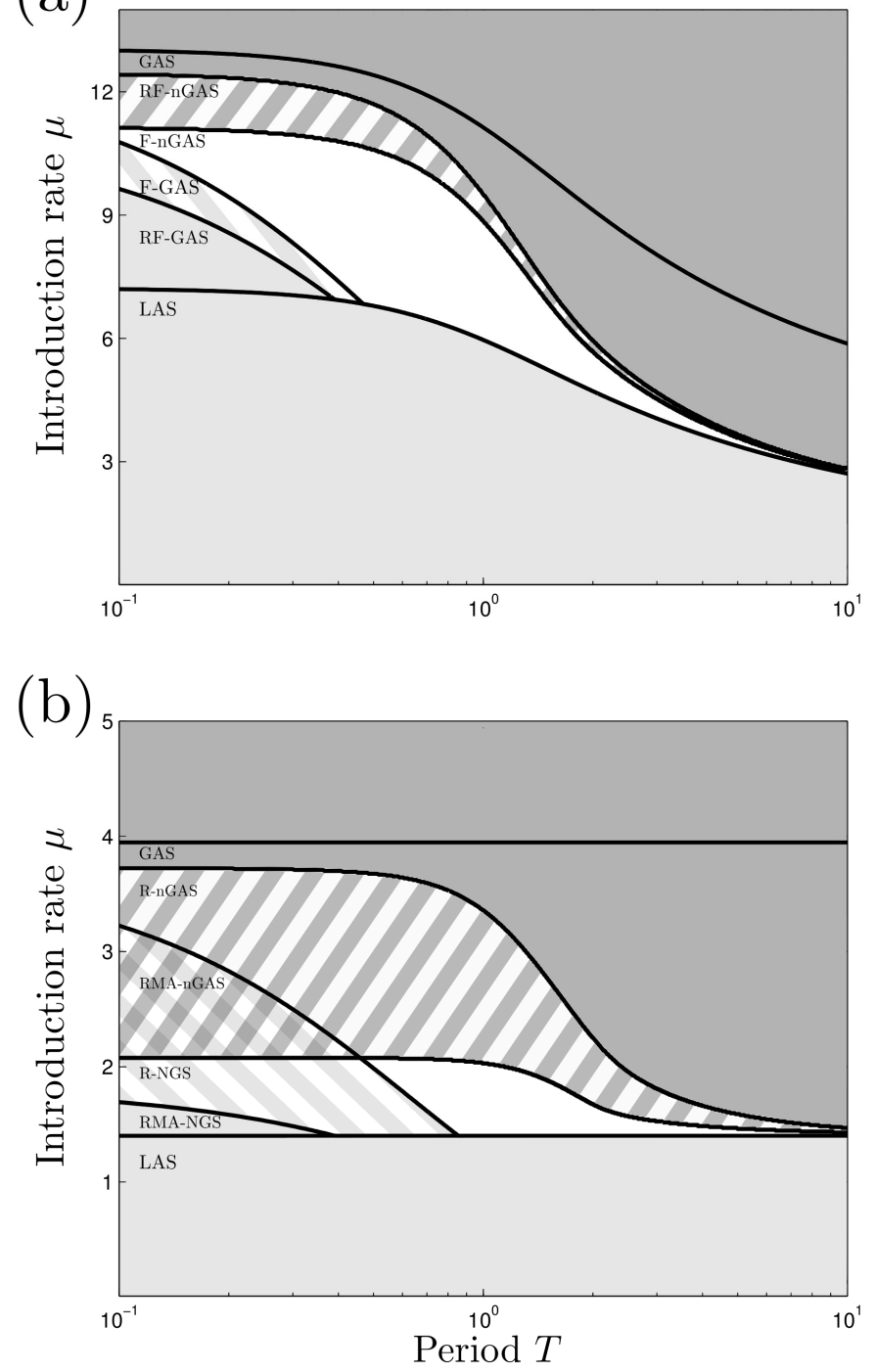

Fig. 1 Stability conditions of pest-free solution as functions of $T$ (in logarithmic scale) and $\mu$. The RFand F- models are compared in (a) while R- and RMA- models are studied in (b). The numerical stability (see text) and NGS regions are represented by dark gray and light gray colors, respectively. In the top dark gray area, both models are analytically GAS while in the bottom one, they are numerically stable. In the dark gray hatched area, only the F-model and RMA-model are numerically stable in (a) and (b), respectively. In the bottom light gray area, both models are unstable while in the top light gray area, they are NGS. In the light gray hatched area, the F- (RMA-) and RF- (R-) models are LAS and NGS in (a) (in (b)), respectively.

In both subfigures, the dark gray areas figure regions where numerical GAS of the pest free solution is achieved for both models. In the area above the GAS curve, 
all models satisfy the sufficient GAS condition. In the dark-gray-hatched region, numerical stability is only achieved for the models without reproductive Allee effect (F-model in Figure 1 a) and the RMA-model in 1(b)). Light gray areas figure regions where global stability is analytically shown not to be achieved, either because the pest-free solution is not LAS (bottom region) or because the NGS condition 27) is satisfied (top region). In the hatched region, the NGS condition is only satisfied for the models with reproductive Allee effect (RF-model in Figure 1(a) and the R-model in 1(b)). In white regions, we have not been able to show NGS analytically, but numerical simulations show that pest free solutions are not GAS. In both figures, the NGS region is larger and numerical stability region smaller for the model with reproductive Allee effect than for the one without. Regarding the NGS condition, this is a direct consequence of the presence of the $\alpha_{r}$ parameter in 39 . This confirms that reproductive Allee effects in the predator population makes pest eradication harder to achieve. Similarly, when comparing both figures, one notices that all curves are at a higher $\mu$-level in the presence of a foraging Allee effect (Figure 11(a)) than without (Figure 1 (b)). This shows that the foraging efficiency Allee effects also hinder pest eradication.

Foraging efficiency Allee effects tend to have a much larger impact than reproductive Allee effect on pest eradication. Indeed, the vertical scale between the two subfigures changes roughly while the difference between curves with and without reproductive Allee effect is not very large. Note that in Figure 1 (1) b), there exists an area where the pest-free solution (7) is numerically stable for the RMA-model while it is analytically not GAS for R-model. In this area, the probability of pest control will be very low if predators are subject to a reproductive Allee effect but pest eradication will be ensured if no Allee effects occur among the predator population: the efficacy of such a given strategy augmentative biological control strategy is entirely determined by the presence or absence of one or more component Allee effects.

An instinctive result illustrated on Figure 1 is that increasing the predator introduction rate $\mu$ helps stability. This result is complemented by the observation that all stability curves separating the regions are decreasing with period $T$ which means that stability is easier to obtain with larger periods. Taking a larger $T$ means that we introduce more predators at each release but less frequently without investing more into predators, which has the tendency to mitigate Allee effects. For instance, in Figure 1.a), taking $\mu=8$ and $T=0.2$ sets us in the top light gray area: the pest-free solution (7) is analytically NGS for both models. However, using the same $\mu$ and $T=1$, solution (7) is then numerically NGS (LAS) for both models (white area) and, with $T=2$, solution (7) is numerically stable (bottom dark gray area). Predators introduction rate has to be large enough to ensure the LAS condition holds: $\mu>\operatorname{arx}_{h} m / b_{0}$. When $\mu$ is smaller, pest eradication can never be achieved. When the introduction rate is intermediate and $\mu$ smaller than the value ensuring numerical stability for $T=0$, applying a small $T$ strategy implies instability or NGS of the pest-free solution while using large $T$ strategies would have ensured GAS of the pest-free solution.

Considering the dark gray hatched region in Figure 1 it seems that the analytic GAS condition becomes less precise with large periods while the LAS condition gives a better approximation of the numerical stability. 
Note that it is not possible, by increasing the period $T$, to pass from a no-LAS situation (bottom light gray area) to a globally stable one (dark gray and dark gray hatched areas) in the Figure 1 (b). Indeed, the horizontality of the LAS condition does not allow to change the stability by only increasing the period $T$ while it is possible to do it when foraging Allee effects occur among predator population. For instance, in Figure 1 (a), taking $\mu=6$ and $T<1$ will lead to a failure while a larger period: $T=2$, will ensure pest eradication

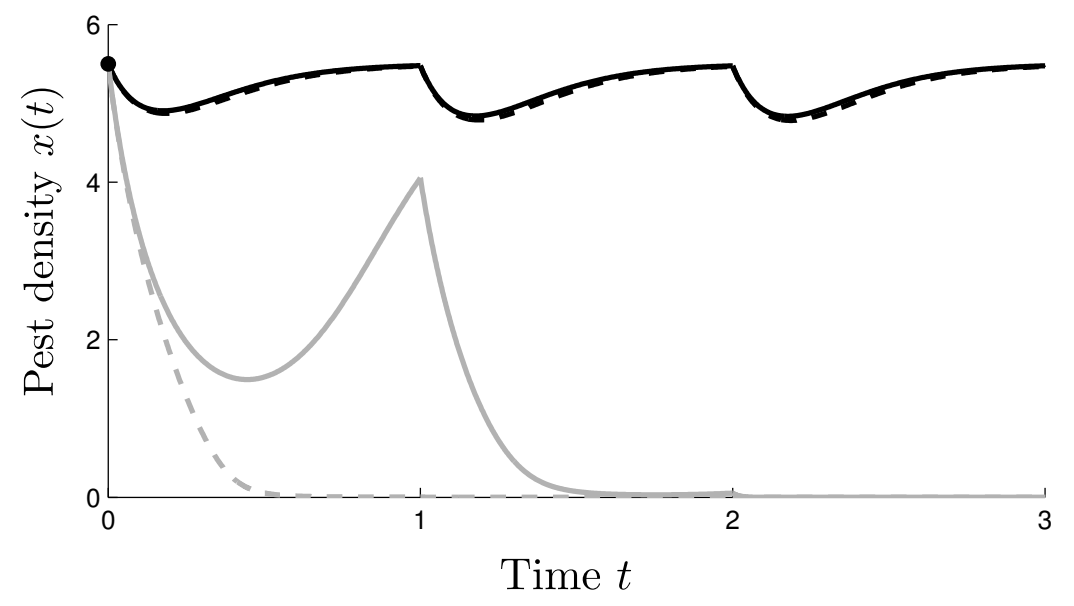

Fig. 2 Comparison of the four models pest-dynamics with $\mu=4, T=1$ and parameters used in Figure 1 Black curves represents pest densities when foraging efficiency Allee effects occur among predator (solid curve: RF-model, dashed curve: F-model). Gray curves represents pest densities of the R-model (solid curve) and RMA-model (dashed curve).

Figure 2 illustrates the dynamics produced by the four models using $\mu=4$ and $T=1$. In this configuration, the pest-free solution is unstable for models with foraging efficiency Allee effects while it is GAS for the R- and RMA- models, see also Figure 11 The pest population is almost eradicated through approximately one(RMAmodel) or two releases (R-model) while it remains at high levels for the RF- and F- models; the pest population stays even very close to the carrying capacity in the two latter cases. Foraging efficiency Allee effects thus strongly influence the efficacy of augmentative biological control, and the release strategies must account for this. To mitigate Allee effects, and in particular foraging Allee effects, large $T$ strategies, implying the release of large batches of predators, should be preferred.

This property is further illustrated in Figure 3 on the R-model. It illustrates that for a fixed $\mu$, increasing the period of release can lead to the success of pest control. Taking $\mu=2.5, T=1.9$ and $(x(0), y(0))=(K, \mu T)$ (solid dark gray area) allows to eradicate pests in approximately three periods while biological control totally fails when $T=0.3$ or $T=0.8$. Note also that the dashed curves describe solutions of the same models, with less pests as initial conditions. The black dashed curve returns to 


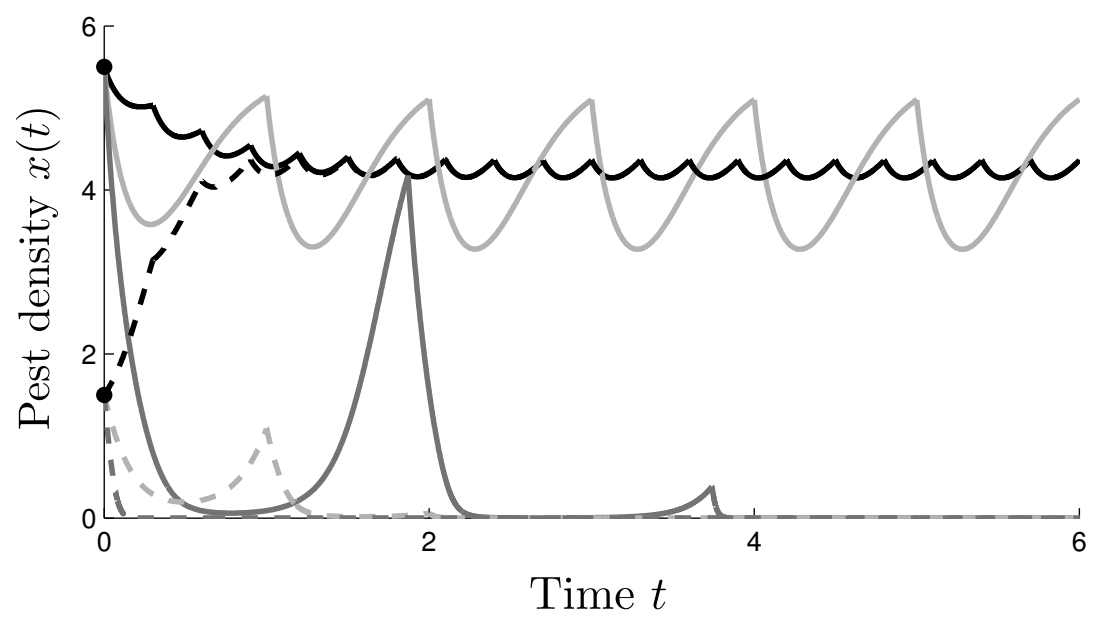

Fig. 3 Influence of period $T$ and initial condition for pest control success on pest dynamics in the Rmodel. Parameters the same than in Figure 1 with $\mu=2.5$. The initial condition used for dashed and solid solutions are $(1.5, \mu T)$ and $(K, \mu T)$, respectively. $T=0.3$ is illustrated in black, $T=0.8$ in light gray and $T=1.9$ in dark gray.

the solid black periodic solution whereas the dashed gray curve converge to the pestfree solution. The light gray curve describe a case when the pest-free solution can be LAS but not GAS since the solid solution represents a failure of pest eradication, while the dashed solution converges to zero.

\section{Discussion}

Intraspecific interactions such as demographic Allee effects have major influences on population dynamics, facilitating or hindering their management (Courchamp et al, 2008; Liebhold and Tobin, 2008). These effects stem from what are called "component" Allee effects. The modelling and analysis of component Allee effects on predators in predator-prey systems has long been overlooked (Bazykin, 1998, Zhou et al 2005), but some recent studies tend to confirm their important impact (Verdy. 2010; Terry, 2015). In this contribution, we proposed a novel mechanistic modelling framework taking into account foraging and/or reproductive component Allee effects for the predator; the genericity of this framework allows it to cover a wide range of biological situations while only imposing biologically relevant constraints on its form. Our model (4) offers a major extension to articles (Verdy, 2010, Terry, 2015) in which only one component Allee effect is taken into account: either the Allee effect modulates the predation rate or the resource assimilation efficiency (in (Verdy, 2010) ), or only reproductive component Allee effects among predators are considered (in (Terry, 2015)). The effects of component Allee effects can then be compared in order to obtain better information about predator-prey dynamics. 
Based on that model, we then developed an augmentative biological control scheme using a semi-discrete approach, where the amount of periodically released predators is determined as the product of a constant release rate and the release period (Mailleret and Grognard, 2009, 2006). Thus, we considered release strategies from small and frequent introductions to rare and large, while still being able to compare them through the value of the release rate.

Stability conditions of a $T$-periodic pest-free stationary solution have been investigated. Although LAS (8) and GAS (9) conditions are strongly correlated with introduction rate $\mu$ and period $T$, the presence or absence of component Allee effects among the natural enemies may seriously influence release strategies. Indeed, we showed that foraging Allee effects impact stability of the pest-free solution more negatively than the reproductive one, limiting more the efficiency of pest control. A foraging Allee effect among the predator population causes a change in the LAS and GAS conditions forms (see also Table 3) and pest eradication is hindered: the level of the theoretical and numerical thresholds for stability in the $(T, \mu)$ plane are higher in Figure 1.a) than in Figure 1 (b). Although the presence of one or both component Allee effects impedes pest eradication, it does not change the qualitative form of the stability region whose upper-boundary is decreasing with period $T$ in each case. Then, considering an introduction rate $\mu$ large enough, pest eradication is ensured for large periods of release even if stability was not achieved for $T$ small. Actually, when a large introduction of predators is done, their quantity allows to overwhelm the Allee effects at least right after the release. This is why rare but large release strategies should be preferred in such biological control programs. Other comparable studies concerning augmentative biological control have been previously carried out. When negative density dependence, i.e. intraspecific competition between predators, occurs among natural enemies, small and frequent release strategies should be favored to ensure stability (Nundloll et al, 2010b a). When, as in the RMA-model, no intraspecific interactions occur among the predator population, even though theoretical stability was not impacted by the release period, (Mailleret and Grognard, 2009, 2006) proposed that small and frequent releases should also be adopted in order to prevent long durations of infestations. The issue appears to be more complicated since the present work recommends the reverse strategy: indeed, the numerical stability curve for the RMA-model in Figure 1(b) is decreasing with period $T$.

We then studied cases where the pest-free solution is LAS but not GAS (NGS) in order to improve our stability knowledge since the GAS condition is only sufficient. In this analysis, we expanded on work in (Terry, 2014a) by optimizing the $y_{m}$-bound and by exploring the impact of different component Allee effects. The NGS condition depends on both the foraging and reproductive component Allee effects (see also Table 3 for observed differences) but the NGS region is limited to smaller $\mu$ values when reproductive mechanisms arise among predators, than when the foraging Allee effect is present. Thus, we have been able to observe cases in which the pest-free solution is numerically globally stable for the RMA-model while it is NGS for the R-model (double hatched area in Figure 1 (b)).

Our theoretical results could help to prevent failures that currently plague augmentative biological control programs. Considering the release rate $\mu$ as a proxy of a budget for practitioners, we suggest to have a large enough period between intro- 
ductions and hence large enough releases to ensure pest eradication. Similarly, large release periods ensure that stability is achieved for smaller release rates. This could then prove to be an economically sensible release strategy. However, as was shown in (Mailleret and Grognard, 2009), too large periods should be avoided since, despite pest eradication being guaranteed, this approach would leave the crop exposed to an ill-timed pest invasion between releases. Indeed, when the period is large, in the absence of pest, the natural enemies population dies out long before the next release so, may an invasion occur, the crop would not be protected so that major damage could happen.

Moreover, we quantified the biological process by studying the asymptotic stability of a periodic pest-free solution of system (6). It means that we considered our system, and its resulting biological control process, over the long-term in Theorem 2 Although augmentative biological control is used for longer duration of pest control than inundative biological control (Lacey et al, 2001), faster convergence might be required in real-life applications in field crops. For that, we considered another approach in Corollary 1 that allows to decrease the pest population level at all times. As expected, restrictions leading to such faster control resulted in a more conservative GAS condition (20) than that obtained in Theorem 2 In practice, augmentative biological control should be based on a balance struck between simple stability requirements and imposing a constant pest decrease.

The techniques used here to study augmentative biological control schemes could be extended to other ecological issues such as reintroductions of species where the objective could be changed to the long-term establishment of predator population. It could for instance be interesting to understand which component Allee effect influences the reintroduction success of a single population in a new environment. Recent investigations have developed tools concerning Integrated Pest management (IPM) strategies, which are the use of different pest control techniques in order to bring and keep the pest level below a certain economic threshold (Elliott et al, 1995, Van Lenteren, 1995). Our impulsive pest control study could bring interesting information for IPM programs, extending theoretical work performed in (Tang et al, 2008 2010, 2013).

This paper considers that periodic releases of predators is a good approximation of augmentative biological control (Terry, 2014a; Wang and Huang, 2015). It is moreover coherent with agronomic practices as described by biologists (Van Lenteren and Bueno, 2003, Bale et al, 2008). Nonetheless, recent papers have investigated statedependent impulsive systems by releasing predators when the pests reach a certain economic threshold (Tang and Cheke, 2005; Jiang and Lu, 2007; Nie et al, 2009, Tang et al, 2010). Such a feedback control could bring relevant improvements over the framework considered in this paper but would require accurate pest measurements which are often not available in practice.

Another interesting topic would be to concentrate on one population but focus more on the Allee effect mechanisms. We could for instance model female and male with two distinct dynamics (Boukal and Berec, 2009) and observe in detail the interaction between reproductive Allee effect linked to difficulties in mate finding and the introduction of individuals. This could help design eradication programs based on the release of sterile males who compete with wild males for females (e.g. SIT tech- 
niques (Dyck et al, 2005). These techniques often require repeated introductions for pest eradication, which is why it would nicely be described using a semi-discrete modeling framework.

Our approach neglects potentially important features of real-life augmentative biological control programs such as the spatial distribution of both populations. However, these programs are most of the time carried out in large culture fields and this requires to know where and how to introduce predators in the space to achieve pest eradication. A study of a patchy environment in which individuals could interact between patches could be considered in future works, as it has been done in (Ghosh et al, 2015). In another direction, considering recent biological control projects, it appears that the quantities of released predators are not exactly the same at each introduction for different reasons: acclimation, environmental variations, quality of breeding before introduction and so on. This is why fixing the quantity of natural enemies may be unrealistic and studying effects of stochastic changes in release sizes could bring valuable information for augmentative biological control. Such questions have for instance been recently explored in (Drury and Lodge, 2009).

\section{Acknowledgments}

The research leading to these results has received funding from the European Union Seventh Framework Programme (FP7/ 2007-2013) under the grant $\mathrm{n}^{\circ} 265865$ (PURE project). This work also benefited from the support of UMT Fiorimed, certified by the French Ministry of Higher Education and Research (2015-2019). This work is part of the PhD thesis of Nicolas Bajeux which is funded by University of Nice Sophia Antipolis. We gratefully thank both reviewers as well as the editors for their relevant and meaningful comments.

\section{References}

Allee WC (1949) Principles of animal ecology. W. B. Saunders Company

Bale J, Van Lenteren J, Bigler F (2008) Biological control and sustainable food production. Philosophical Transactions of the Royal Society of London B: Biological Sciences 363(1492):761-776

Bazykin AD (1998) Nonlinear dynamics of interacting populations, vol 11. World Scientific

Berec L, Angulo E, Courchamp F (2007) Multiple allee effects and population management. Trends in Ecology \& Evolution 22(4):185-191

Bolognesi C, Morasso G (2000) Genotoxicity of pesticides: potential risk for consumers. Trends in Food Science \& Technology 11(4):182-187

Bompard A, Amat I, Fauvergue X, Spataro T (2013) Host-parasitoid dynamics and the success of biological control when parasitoids are prone to allee effects. PLoS ONE 8:e76,768, DOI 10.1371/journal.pone.0076768

Boukal DS, Berec L (2009) Modelling mate-finding allee effects and populations dynamics, with applications in pest control. Population Ecology 51(3):445-458 
Collier T, Steenwyk R (2004) A critical evaluation of augmentative biological control. Biological Control 31(2):245-256

Courchamp F, Macdonald DW (2001) Crucial importance of pack size in the african wild dog lycaon pictus. Animal Conservation 4(02):169-174

Courchamp F, Berec L, Gascoigne J (2008) Allee effects in ecology and conservation. Oxford University Press

DeBach P, Rosen D (1991) Biological control by natural enemies. CUP Archive

Derederec A, Courchamp F (2007) Importance of the allee effect for reintroductions. Écoscience 14:440-451

Drury KL, Lodge DM (2009) Using mean first passage times to quantify equilibrium resilience in perturbed intraguild predation systems. Theoretical Ecology 2(1):4151

Dyck VA, Hendrichs J, Robinson AS (2005) Sterile insect technique. Springer

Elliott N, Farrell J, Gutierrez A, van Lenteren JC, Walton M, Wratten S, Dent D (1995) Integrated pest management. Springer Science \& Business Media

Fauvergue X, Hopper KR (2009) French wasps in the new world: experimental biological control introductions reveal a demographic allee effect. Population Ecology 51:385-397

Floquet G (1883) Sur les équations différentielles linéaires à coefficients périodiques. In: Annales scientifiques de l'École normale supérieure, vol 12, pp 47-88

Gascoigne J, Berec L, Gregory S, Courchamp F (2009) Dangerously few liaisons: a review of mate-finding allee effects. Population Ecology 51(3):355-372

Ghosh B, Grognard F, Mailleret L (2015) Natural enemies deployment in patchy environments for augmentative biological control. Applied Mathematics and Computation 266:982-999

Holling CS (1959) The components of predation as revealed by a study of smallmammal predation of the european pine sawfly. The Canadian Entomolgist 91:293-320

Jiang G, Lu Q (2007) Impulsive state feedback control of a predator-prey model. Journal of Computational and Applied Mathematics 200(1):193-207

Khalil HK, Grizzle J (2002) Nonlinear systems, vol 3. Prentice hall Upper Saddle River

Lacey L, Frutos R, Kaya H, Vail P (2001) Insect pathogens as biological control agents: do they have a future? Biological control 21(3):230-248

Lakshmikantham V, Bainov D, Simeonov PS (1989) Theory of impulsive differential equations, vol 6 . World scientific

Liebhold AM, Tobin PC (2008) Population ecology of insect invasions and their management. Annu Rev Entomol 53:387-408

Lu ZH, Chi XB, Chen LS (2003) Impulsive control strategies in biological control of pesticide. Theoretical Population Biology 64(1):39-47

Mailleret L, Grognard F (2006) Optimal release policy for prophylactic biological control. Positive systems pp 89-96

Mailleret L, Grognard F (2009) Global stability and optimisation of a general impulsive biological control model. Mathematical Biosciences 221:91-100

Mailleret L, Lemesle V (2009) A note on semi-discrete modelling in the life sciences. Philosophical Transactions of the Royal Society A-Mathematical Physical 
and Engineering Sciences 367:2113-2138

MATLAB (2012) version 8.0.0 (R2012b). The MathWorks Inc., Natick, Massachusetts, United States

McLaughlin A, Mineau P (1995) The impact of agricultural practices on biodiversity. Agriculture, Ecosystems \& Environment 55(3):201-212

Møller AP, Gregersen J (1994) Sexual selection and the barn swallow, vol 8. Oxford University Press Oxford

Møller AP, Legendre S (2001) Allee effect, sexual selection and demographic stochasticity. Oikos 92(1):27-34

Nie L, Teng Z, Hu L, Peng J (2009) Existence and stability of periodic solution of a predator-prey model with state-dependent impulsive effects. Mathematics and Computers in Simulation 79(7):2122-2134

Nundloll S, Mailleret L, Grognard F (2010a) Influence of intrapredatory interferences on impulsive biological control efficiency. Bulletin of mathematical biology 72(8):2113-2138

Nundloll S, Mailleret L, Grognard F (2010b) Two models of interfering predators in impulsive biological control. Journal of Biological Dynamics 4(1):102-114

Oerke EC (2006) Crop losses to pests. The Journal of Agricultural Science 144(01):31-43

Pimentel D, Edwards CA (1982) Pesticides and ecosystems. BioScience 32(7):595600

Pimentel D, Acquay H, Biltonen M, Rice P, Silva M, Nelson J, Lipner V, Giordano S, Horowitz A, D'amore M (1992) Environmental and economic costs of pesticide use. BioScience pp 750-760

Rosenzweig ML, MacArthur RH (1963) Graphical representation and stability conditions of predator-prey interactions. American Naturalist 97(895):209-223

Simberloff D (2009) The role of propagule pressure in biological invasions. Annual Review of Ecology, Evolution, and Systematics 40:81-102

Stephens PA, Sutherland WJ (1999) Consequences of the allee effect for behaviour, ecology and conservation. Trends in Ecology \& Evolution 14(10):401-405

Tang S, Cheke RA (2005) State-dependent impulsive models of integrated pest management (ipm) strategies and their dynamic consequences. Journal of Mathematical Biology 50(3):257-292

Tang S, Xiao Y, Cheke RA (2008) Multiple attractors of host-parasitoid models with integrated pest management strategies: Eradication, persistence and outbreak. Theoretical Population Biology 73(2):181-197

Tang S, Tang G, Cheke RA (2010) Optimum timing for integrated pest management: modelling rates of pesticide application and natural enemy releases. Journal of Theoretical Biology 264(2):623-638

Tang S, Liang J, Tan Y, Cheke RA (2013) Threshold conditions for integrated pest management models with pesticides that have residual effects. Journal of mathematical biology 66(1-2):1-35

Terry AJ (2014a) Biocontrol in an impulsive predator-prey model. Mathematical biosciences $256: 102-115$

Terry AJ (2014b) A predator-prey model with generic birth and death rates for the predator. Mathematical biosciences 248:57-66 
Terry AJ (2015) Predator-prey models with component allee effect for predator reproduction. Journal of Mathematical Biology pp 1-28

Van Lenteren J (1995) Integrated pest management in protected crops. In: Dent D (ed) Integrated pest management, Chapman \& Hall, London, pp 311-343

Van Lenteren J (2000) Success in biological control of arthropods by augmentation of natural enemies. In: Biological control: Measures of success, Springer, pp 77-103

Van Lenteren JC, Bueno VH (2003) Augmentative biological control of arthropods in latin america. BioControl 48(2):123-139

Verdy A (2010) Modulation of predator-prey interactions by the Allee effect. Ecological Modelling 221:1098-1107

Wang S, Huang Q (2015) Bifurcation of nontrivial periodic solutions for a beddington-deangelis interference model with impulsive biological control. Applied Mathematical Modelling 39(5):1470-1479

Wilson C, Tisdell C (2001) Why farmers continue to use pesticides despite environmental, health and sustainability costs. Ecological economics 39(3):449-462

Wittmann MJ, Metzler D, Gabriel W, Jeschke JM (2014) Decomposing propagule pressure: the effects of propagule size and propagule frequency on invasion success. Oikos 123(4):441-450

Zhang H, Georgescu P, Chen L (2008) On the impulsive controllability and bifurcation of a predator-pest model of ipm. BioSystems 93(3):151-171

Zhou SR, Liu YF, Wang G (2005) The stability of predator-prey systems subject to the allee effects. Theoretical Population Biology 67:23 - 31 